Pacific Journal of Mathematics

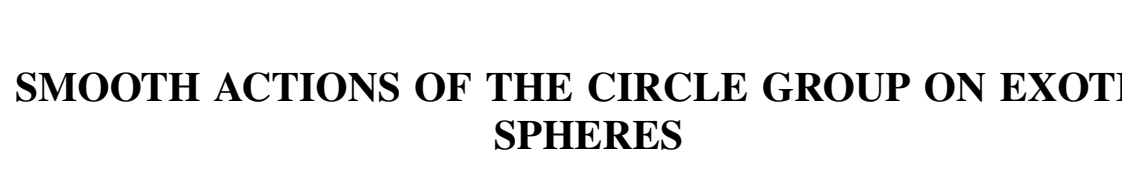




\title{
SMOOTH ACTIONS OF THE CIRCLE GROUP ON EXOTIC SPHERES
}

\author{
VAPPALA J. JOSEPH
}

\begin{abstract}
Recent work of Schultz translates the question of which exotic spheres $S^{n}$ admit semifree circle actions with $k$-dimensional fixed point set entirely to problems in homotopy theory provided the spheres bound spin manifolds. In this article we study circle actions on homotopy spheres not bounding spin manifolds and prove, in particular, that the spin boundary hypothesis can be dropped if $(n-k)$ is not divisible by 128. It is also proved that any ordinary sphere can be realized as the fixed point set of such a circle action on a homotopy sphere which is not a spin boundary; some of these actions are not necessarily semi-free. This extends earlier results obtained by Bredon and Schultz. The Adams conjecture, its consequences regarding splittings of certain classifying spaces and standard results of simply-connected surgery are used to construct the actions. The computations involved relate to showing that certain surgery obstructions vanish.
\end{abstract}

1. Introduction. Results due to Schultz give a purely homotopy theoretic characterization of those homotopy $(n+2 k)$-spheres admitting smooth semi-free circle actions with $n$-dimensional fixed point sets provided one limits attention to exotic spheres bounding spin manifolds. The method of proof is similar to that described in [14] for actions of prime order cyclic groups; a detailed account will appear in [21]. Since the premise of this article relates directly to [21], we outline some of the results contained there.

Given nonnegative integers $m<n$, let $C P_{m}^{n}$ denote the quotient complex $C P^{n} / C P^{m}$. $C P_{m}^{n}$ is also the Thom space of $m$ copies of the canonical line bundle over $C P^{n-m}[10,11]$. For some integer $A$ depending only on $n-m$, the complexes $\sum^{A} C P_{m}^{n}$ and $C P_{m+A}^{n+A}$ are stably equivalent [4]. Using this periodicity one can define a spectrum (finite) $C P_{m}^{n}$ for all integers $m<n$. In the same manner, it is also possible to form a limit spectrum $C P_{m}^{\infty}$ for all finite integers $m$. If $m$ and $n$ are positive integers, there are elementary coexact sequences of the form

$$
S^{2 m-2} \longrightarrow C P_{m-1}^{n} \longrightarrow C P_{m}^{n} \stackrel{\delta}{\longrightarrow} S^{2 m-1} ;
$$

and by periodicity and limit arguments there are similar sequences when $m$ and $n$ are arbitrary integers or $n=\infty$.

Let $F_{S^{1}}\left(C^{q}\right)$ be the topological monoid of $S^{1}$ equivariant self-maps 
of the unit sphere $S^{2 q-1}$ contained in $C^{q}$ (action via scalar multiplication), and let $F_{S^{1}}$ be the corresponding stabilization. There are natural inclusions of the unitary group $U_{q}$ in $F_{S_{1}}\left(C^{q}\right)$ and of $U=$ $\lim U_{q}$ in $F_{S^{1}}$, and therefore one has quotient spaces and a stabilization map of the form $\sigma: F_{S^{1}}\left(C^{q}\right) / U_{q} \rightarrow F_{S^{1}} / U$. From [9], we know that the homotopy groups of $F_{S^{1}}$ are isomorphic to those of the free infinite loop space $\Omega^{\infty} \Sigma^{\infty}\left(\Sigma C P_{0}^{\infty}\right)$. Now we can state the following:

THEOREM 1.1 [21]. Let $S_{r}^{n+2 k}$ be a homotopy ( $\left.n+2 k\right)$-sphere bounding a spin manifold, and let $P(\gamma) \subseteq \Pi_{n+2 k}^{S}$ be its PontryaginThom invariant. Then there is a smooth semi-free $S^{1}$ action on $S_{r}^{n+2 k}$ with n-dimensional fixed point set if and only if:

(1) There is a class $\beta \in \Pi_{n}^{S}\left(\Sigma C P_{-k}^{\infty}\right) \cong \Pi_{n+2 k}^{S}\left(\Sigma^{2 k+1} C P_{-k}^{\infty}\right)$ such that $\delta_{*} \Sigma^{2 k} \beta \varepsilon P(\gamma) \subseteq \Pi_{n+2 k}^{S}$.

(2) There is a class $\alpha \varepsilon \Pi_{n}\left(F_{S^{1}}\left(C^{k}\right) / U_{k}\right)$ such that $\sigma_{*} \alpha$ equals the image of $\beta$ under the composition

$$
\Pi_{n}^{S}\left(\Sigma C P_{-k}^{\infty}\right) \stackrel{K_{*}}{\longrightarrow} \Pi_{n}^{S}\left(\Sigma C P_{0}^{\infty}\right) \cong \Pi_{n}\left(F_{S^{1}}\right) \longrightarrow \Pi_{n}\left(F_{S^{1}} / U\right),
$$

in which $K$ is formally the map collapsing the subspectrum $\Sigma C P_{-k}^{-1}$ to a point.

If $b \operatorname{Spin}_{m+1}$ is the group of homotopy $m$-spheres bounding spin manifolds, $\theta_{m} / b \operatorname{Spin}_{m+1}=Z_{2}$ if $m=1,2 \bmod 8, m>8$ and zero otherwise [5]. Therefore the question of which homotopy spheres, not necessarily in $b \mathrm{Spin}_{m+1}$, admit semi-free circle actions as in the theorem reduces to whether any such actions at all exist on exotic spheres not bounding spin manifolds of the appropriate dimensions. We list below the known results and the results obtained in this article. [15].

(1) If $k$ is odd no such examples exist by results of Schultz

(2) If $n$ and $k$ are both even, Bredon has examples [6].

(3) If $n$ is odd and $k \neq 0$ (8), $k>2$ being even, Schultz has constructed examples [16]. In general, the problem of finding examples with $n$ odd and $k$ even reduces to the following problem about vector bundles:

Problem. Suppose we are given an odd integer $n$ and an integer $k$ such that $n+2 k=8 s+1>8$. Is there a real vector bundle $\zeta$ over $S^{n+1} \times C P^{k-1}$, which is stably trivial over $S^{n+1}$ and $C P^{k-1}$ such that the Pontryagin classes of $\psi^{3} \zeta-\zeta$ satisfy

$$
\begin{gathered}
\left\langle\hat{A}_{28}\left(\psi^{3} \zeta-\zeta\right),\left[S^{n+2} \times C P^{k-1}\right]\right\rangle \varepsilon 2 Z+1 ;\left\langle L_{2 s}\left(\psi^{3 \zeta}-\zeta\right),\right. \\
\left.\left[S^{n+2} \times C P^{k-1}\right]\right\rangle=0 ?
\end{gathered}
$$


We discuss this reduction in $\S 2$ and show how a positive solution leads to circle actions. In $\S 3$, the problem is answered positively for $k \neq 0(64)$.

(4) If $n$ is odd and $k=2$, then no examples exist according to Levine [12]. In this case the methods of this paper can be adapted to yield actions which are nearly semi-free. These actions, discussed in $\S 2$, have three types of orbits; namely fixed points, free orbits and orbits of type $Z_{2 n}$.

2. Fixed point sets of co-dimension $2 \bmod 4$. We study smooth effective circle group actions on homotopy spheres with standard spheres as fixed point sets. The homotopy spheres do not bound spin manifolds and the fixed point sets have co-dimension 2 mod 4. It is known that such actions cannot be semi-free [15]. The actions we construct have three types of orbits: fixed points, free orbits and orbits of type $Z_{2 n}$ for given $n$.

THEOREM 2.1. Let $k>0$. There is a smooth effective action of the circle group on a homotopy $(8 k+1)$-sphere not bounding a spin manifold with fixed point set a standard sphere of co-dimen$\operatorname{sion} 4 q+2, q>1$.

We prove this result by constructing a homotopy sphere $\Sigma^{8 k+1}$ and an action on it with the desired properties. In order to do this, it is necessary to show that certain normal maps into the space $M=S^{8 k-4 q+2} \times{ }_{S^{1}} S^{4 q-1}$ are normally co-bordant to homotopy equivalences. Here, $M$ is the orbit space of the $S^{1}$ action (trivial $\left.\bigoplus \psi^{2 n} C\right) \times(2 q C)$ on $S^{8 k-4 q+2} \times S^{4 q-1} . \quad 2 q C$ is the standard free action of $S^{1}$ on $S^{4 q-1} ; \psi^{2 n} C$ is given by $z z_{1}=z^{2 n} z_{1}$ for $z$ in $S^{1}$ and $z_{1}$ in $C$. Since $M$ is a simply connected $8 k$-dimensional manifold, the obstruction to finding a normal cobordism of a normal map to a homotopy equivalence is an index difference. The normal maps we consider are obtained via fiber homotopically trivial bundles over $M$. Hence, it is sufficient, by Hirzebruch's index theorem, to calculate the rational Pontryagin classes of fiber homotopically trivial bundles over $M$. All such bundles arise from elements of $[M, F / 0]$, [7]. For our purposes it is enough to consider elements of $[M, F / 0]$ obtained from a simpler set as pullbacks in the following manner:

$$
\begin{aligned}
{\left[S^{8 k-4 q}\left(C P^{2 q-1}\right)^{\eta^{2 n}}, F / 0\right]=} & {\left[\left(C P^{2 q-1}\right)^{\text {trivial }} \bigoplus^{\eta^{2 n}}, F / 0\right] } \\
= & {\left[D^{8 k-4 q+2} \times{ }_{S^{1}} S^{4 q-1} / S^{8 k-4 q+1} \times{ }_{S^{1}} S^{4 q-1}, F / 0\right] } \\
= & {\left[S^{8 k-4 q+2} \times{ }_{S^{1}} S^{4 q-1} / D^{8 k-4 q+1} \times{ }_{S^{1}} S^{4 q-1}, F / 0\right] } \\
& \mid(\text { Collapse }) \\
& {[M, F / 0] . }
\end{aligned}
$$


Here $\eta$ is the Hopf bundle $S^{4 q-1} \rightarrow C P^{2 q-1}$ so that $\eta^{2 n}$ is the bundle

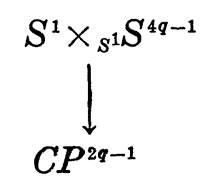

where $S^{1} \times{ }_{S^{1}} S^{4 q-1}$ is the orbit space of the action $\psi^{2 n} C \times 2 q C$ on $S^{1} \times S^{4 q-1}$. $D_{-}^{n}$ denotes the lower hemisphere of $S^{n}$. This means that we are, in fact, interested in studying bundles over the space $S^{8 k-4 q}\left(C P^{2 q-1}\right)^{\eta^{2 n}}$.

Bott periodicity and Thom isomorphisms give all the information we need for calculating the rational Pontryagin classes in this case. We denote the Bott periodicity isomorphism by $\beta$, the $K$-theory Thom isomorphism $K^{*}\left(C P^{2 q-1}\right) \rightarrow K\left(\left(C P^{2 q-1}\right)^{\eta^{2 n}}\right)$ by Th. We also let $\mu=\eta-1$ in $K^{*}\left(C P^{n}\right)$. Then $\mu$ generates $K^{*}\left(C P^{n}\right)$ multiplicatively [4]. Let $\psi_{R}^{3}$ denote an Adams' operation in real $K$-theory. With these notations, we can state the following:

LEMmA 2.2. Let $\zeta=\left[\beta^{4 k-2 q} \operatorname{Th}\left(\mu^{2 q-1}\right)\right]$. Then $\zeta=\sigma \otimes C$, where $\sigma$ belongs to $K O\left[S^{8 k-4 q}\left(C P^{2 q-1}\right)\right]$ and $\eta^{2 n}$ is the pullback of the generator of $K O\left(S^{8 k}\right)$ under the collapsing map

$$
\begin{aligned}
\bigsqcup^{8 k-4 q}\left(C P^{2 q-1}\right)^{\eta^{2 n}} & \\
S^{8 k-4 q}\left(C P^{2 q-1} / C P^{2 q-2}\right)^{\eta^{2 n}} & =S^{8 k-4 q}\left(S^{4 q-2}\right)^{\eta^{2 n}} . \\
& =S^{8 k}
\end{aligned}
$$

Let $\zeta^{\prime \prime}=\beta^{4 k-2 q} \mathrm{Th}\left(\mu^{2 q-3}\right), \zeta^{\prime}=r e \zeta^{\prime \prime}$, where $r e: K \rightarrow K O$ is realification

$$
\alpha=\psi_{R}^{3} \zeta^{\prime}-\zeta^{\prime}, \gamma=\psi_{R}^{3} \sigma-\sigma .
$$

$3^{e} \alpha$ and $3^{e} \gamma$ have fiberhomotopically trivial associated sphere bundles by Adams conjecture [13]. We denote the pullbacks of $\alpha, \gamma$ to $M$ by the same symbols. The computations necessary to prove (2.1) are contained in the following

LEMMA 2.3. There is an integer pair $(x, y)$ such that $y$ is odd and $\left\langle L_{2 k}\left(\tau_{M} \oplus 3^{e} x \alpha+3^{e} y \gamma\right),[M]\right\rangle=\left\langle L_{2 k}\left(\tau_{M}\right),[M]\right\rangle$. Here $L_{2 k}$ denotes the Hirzebruch $L$ class and $\tau_{M}$ is the tangent bundle of $M .{ }^{[M]}$ is the fundamental class of $M$.

Proofs of Lemmas 2.2 and 2.3 are postponed.

Proof of Theorem 2.1. The elements $3^{e} \alpha$ and $3^{e} \gamma$ lift cannonically 
to $[M, F / 0]$ for large $e$. Therefore, for any pair of integers $(x, y)$ $3^{e} x \alpha \oplus 3^{e} y \gamma$ gives a normal map $f_{x, y}: X^{8 k} \rightarrow M$ so that

$$
\tau_{x}+f^{*} \gamma_{M L}=f^{*}\left(3^{e} x \alpha \oplus 3^{e} y \gamma\right)[7] \text {. }
$$

Here $\gamma_{M}$ is the stable normal bundle of $M$. Since the bundle $3^{e} x \alpha \oplus$ $3^{e} y \gamma$ is induced from

$\left[S^{8 k-4 q+2} \times{ }_{S^{1}} S^{4 q-1} / D_{-}^{8 k-4 q+2} \times{ }_{S^{1}} S^{4 q-1}, F / 0\right]$, we may choose $f$ so that $f_{x, y}$ restricted to $f_{x, y}^{-1}\left(D_{-}^{8 k-4 q+2} \times{ }_{S^{1}} S^{4 q-1}\right)$ is a diffeomorphism. Excise the open manifold over which $f$ is a diffeomorphism. We get a relative map of pairs

$$
f_{x, y}^{\prime}:(X, \partial X) \longrightarrow\left(D^{8 k-4 q+2}, S^{8 k-4 q+1}\right) X_{S^{1}} S^{4 q-1}
$$

such that $f^{\prime} / \partial X^{\prime}$ is a diffeomorphism.

The relative surgery obstruction of $f^{\prime}$ is the same as the surgery obstruction of $f$ since all the spaces involved are simply connected [20]. So $f^{\prime}$ is normally cobordant to a homotopy equivalence which is a diffeomorphism on the boundary if and only if the surgery obstruction of $f$ is 0 . That is,

$$
1 / 8[\text { Index } X \text {-Index } M]=0 \text {. }
$$

But Index $X=\left\langle L_{2 k}\left(\tau_{X}\right),[X]\right\rangle$

$=\left\langle L_{2 k}\left(f^{*}\left(\tau_{M} \oplus 3^{e} x \alpha \oplus 3^{e} y \gamma\right)\right),[X]\right\rangle$

$=\left\langle L_{2 k}\left(\tau_{M} \oplus 3^{e} x \alpha \oplus 3^{e} y \gamma\right),[X]\right\rangle$

$=\left\langle L_{2 k}\left(\tau_{M} \oplus 3^{e} x \alpha \oplus 3^{e} y \gamma\right),[M]\right\rangle$

$=$ Index $M$, for some choice of $(x, y), y$ odd

such that

$$
\left.f_{x, y}^{\prime}:(X, \partial X) \longrightarrow\left(D^{8 k-4 q+2}, S^{8 k-4 q+1}\right) \times{ }_{S^{1}} S^{4 q-1}\right)
$$

is normally cobordant to a homotopy equivalence, which is a diffeomorphism on the boundary. Consider the pullback diagram

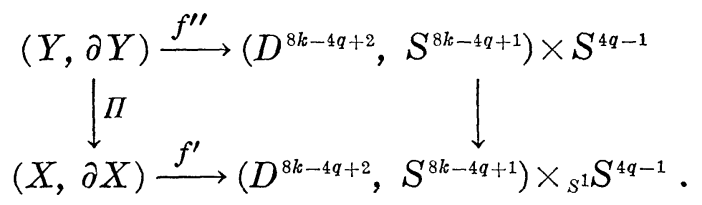

The orbit map $I$ is a principal bundle projection, since the action (trivial $\left.\oplus \psi^{2 n} C\right) \times 2 q C$ is free. So there is a free action on $(Y, \partial Y)$ such that $f^{\prime \prime}$ is an $S^{1}$ equivariant homotopy equivalence. Further, $f^{\prime \prime} / \partial Y$ is a diffeomorphism. Let $\sum_{(x, y)}^{8 k+1}=Y \bigcup_{f^{\prime \prime} / \partial Y} S^{8 k-4 q+1} \times D^{4 q}$. Then $\sum_{(x, y)}^{8 k+1}$ has a smooth effective circle action given by the circle actions on the two component pieces. Clearly, the fixed point set is $4 q+2$. 
By a Mayer-Vietoris argument $\sum_{(x, y)}^{8 k+1}$ is a homology sphere. It is simply connected by van Kampen's theorem. Hence $\sum_{(x, y)}^{8 k+1}$ is a homotopy $(8 k+1)$-dimensional sphere.

It remains to show that this sphere does not bound a spin manifold. This is done by identifying the Pontryagin-Thom invariant of $\sum_{(x, y)}^{8 k+1}$.

Consider the map on Thom spaces obtained via the following diagram of bundle maps:

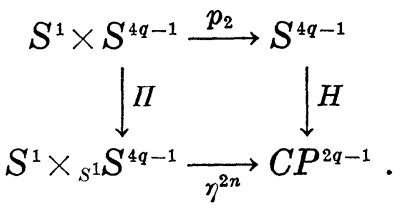

Here $I$ is the orbit map for the action $\psi^{2 n} C \times 2 q C, H$ is the Hopf map and $p_{2}$ is the projection. Let $X_{k, q}=S^{8 k-4 q}\left(C P^{2 q-1}\right)$ and $\Pi^{*}$ : $\left[X_{k, q}, F / 0_{(2)}\right] \rightarrow\left[S^{8 k-4 q+2}\left(S^{4 q-1} V S^{0}\right), \quad F / 0_{(2)}\right]$ the map induced by this Thom space map. Here $X_{(p)}$ is the localization of $X$ at $p[18,19]$.

We wish to calculate $\Pi^{*}\left(3^{e} x \alpha \oplus 3^{e} y \gamma\right)$. It is enough to calculate the image of $3^{e}\left(x \zeta^{\prime}+y \sigma\right)$ under the map analogous to the above with $\mathrm{BSO}_{(2)}$ replacing $F / O_{(2)}$.

$$
\begin{aligned}
{\left[S^{8 k-4 q+2}\left(S^{4 q-1} V S^{0}\right), \mathrm{BSO}_{(2)}\right] } & \left.=\Pi_{8 k+1}\left(\mathrm{BSO}_{(2)}\right) \times \Pi_{8 k-4 q+2}\left(\mathrm{BSO}_{(2)}\right)\right] \\
\text { (by Bott periodicity) } & =\left[\Pi_{8 k+1} \mathrm{BSO} \otimes Z_{(2)}\right] \times\left[\Pi_{8 k-4 q+2}(\mathrm{BSO})\right] \\
& \left.\otimes Z_{(2)}\right] .
\end{aligned}
$$

So the group has exponent two. Therefore, the factor $3^{e}$ acts as identity and may be suppressed. The bundle $\zeta$ has a complex structure; in fact $\zeta^{\prime}=r e \zeta^{\prime \prime}$ where $\zeta^{\prime \prime}=\beta^{4 k-2 q} \mathrm{Th}\left(\mu^{2 q-3}\right)$. Since $H^{*}=0$ on $K\left(C P^{2 q-1}\right)$, it follows by naturality of Thom isomorphism and the Bott periodicity that

$$
\Pi^{*} \zeta^{\prime \prime}=0 \text { and hence } \Pi^{*} \zeta^{\prime}=0 .
$$

By Lemma 2.2, $\sigma$ is the pullback of the generator of $\Pi_{8 k}(\mathrm{BSO})$ under the collapsing map

$$
S^{8 k-4 q}\left(C P^{2 q-1}\right)^{\eta^{2 n}} \longrightarrow S^{8 k-4 q}\left(C P^{2 q-1} / C P^{2 q-2}\right)^{\eta^{2 n}}=S^{8 k} .
$$

By definition of $\Pi^{*}$, it follows that $\Pi^{*} \sigma$ is the composite of this generator with the map

$$
S^{8 k-4 q}\left(S^{4 q-1}\right)^{\text {trivial }} \longrightarrow S^{8 k-4 q}\left(C P^{2 q-1}\right)^{\gamma^{2 n}}
$$

induced by the suspended Hopf map $H$ on the Thom spaces. Looking at this map on the top cells, it is seen that $\Pi^{*}$ has the form $M_{k} \eta$ and therefore 


$$
\Pi^{*}\left(3^{e} x \alpha \oplus 3^{e} y \gamma\right)=M_{k} \eta \text { since } y \text { is odd. }
$$

Elementary attaching considerations $[17,20]$ tell us that $\Pi^{*}\left(3^{e} x \alpha \oplus 3^{e} y y\right)$ is the Pontryagin-Thom invariant of $\sum_{(x, y)}^{8 k+1}$. By the results in $\S 1$ and (2.4) and (2.5) this completes our proof.

Now, we return to the proofs of computational Lemmas 2.2-2.3.

Proof of Lemma 2.2. Look at the following diagram

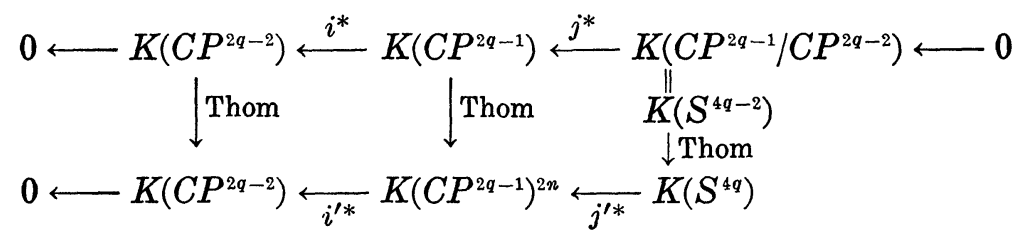

where $i$ is the inclusion and $j$ is the collapse. $j^{*}$ is a split monomorphism identifying the generator of $K\left(S^{4 q-2}\right)$ with $\mu^{2 q-1}$. $\left(j^{\prime}\right)^{*}$ identifies the generator $K\left(S^{4 q}\right)$ with $\mathrm{Th}\left(\mu^{2 q-1}\right) \eta^{2 n}$. Suspend this diagram $(8 k-4 q)$ times and look at the map of the corresponding $K O$ groups into the above diagram under the complexification map. Lemma 2.2 is now obvious.

Proof of Lemma 2.3.

$$
\begin{array}{r}
\alpha=\psi_{R}^{3} r e \zeta^{\prime \prime}-r e \zeta^{\prime \prime}, \gamma=\psi_{R}^{3} \sigma-\sigma, \\
\sigma \times C=\zeta . \quad \text { Also } \zeta=\beta^{4 k-2 q} \operatorname{Th}\left(\mu^{2 q-1}\right) \\
\zeta^{\prime \prime}=\beta^{4 k-2 q} \operatorname{Th}\left(\mu^{2 q-3}\right) .
\end{array}
$$

We can calculate $\operatorname{ch}(\quad)$ and $\operatorname{ch}(\quad)$ as follows;

$$
\begin{aligned}
& \operatorname{ch} \operatorname{Th}\left(\mu^{2 q-1}\right)=\operatorname{ch}\left(\mu^{2 q-1}\right) \cup\left[\operatorname{Td}\left(\eta^{2 n}\right)\right]^{-1} \cdot U^{\eta^{2 n}} \\
& \operatorname{ch} \operatorname{Th}\left(\mu^{2 q-3}\right)=\operatorname{ch}\left(\mu^{2 q-3}\right) \cup\left[\operatorname{Td}\left(\eta^{2 n}\right)\right]^{-1} \cdot U^{\eta^{2 n}} .
\end{aligned}
$$

We have $\operatorname{ch}(\mu)=\operatorname{ch}(\eta-1)=e^{c}-1$, where $c$ is the generator of $H^{2}\left(C P^{2 q-1}\right)$, [4]. Hence $\operatorname{ch}\left(\mu^{2 q-1}\right)=c^{2 q-1}$. Further $\mathrm{Td}\left(\eta^{2 n}\right)=Q\left[c_{1}\left(\eta^{2 n}\right)\right]$ where $Q(x)=x / 1-e^{-x}$. So $\left[\operatorname{Td}\left(\eta^{2 n}\right)\right]^{-1}=1-e^{-x} / x$ and $x=c_{1}\left(\eta^{2 n}\right)=$ $X\left(\eta^{2 n}\right)=2 n c$. (Here $X$ denotes the Euler class.) Therefore

$$
\begin{aligned}
& \operatorname{Td}\left[\left(\eta^{2 n}\right)\right]^{-1}=1-c d+2 n^{2} \frac{c^{2}}{3}+\cdots \\
& \operatorname{ch} \operatorname{Th}\left(\mu^{2 q-1}\right)=c^{2 q-1}
\end{aligned}
$$

$$
\operatorname{ch} \operatorname{Th}\left(\mu^{2 q-3}\right)=c^{2 q-3}\left[1+A c+B c^{2}\right] . \quad\left[1-n c+\frac{2}{3} n^{2} c^{2}\right]
$$

where $A=2 q-3 / 2, B=2 q-3 / 6+(2 q-3)(2 q-4) / 8$ 


$$
\operatorname{ch} \operatorname{Th}\left(\mu^{2 q-3}\right)=c^{2 q-3}\left[1+A^{\prime} c+\left(\frac{2}{3} n^{2}-n A+B\right) c^{2}\right] .
$$

We don't need $A^{\prime}$ for further calculations.

$$
\begin{aligned}
\frac{2}{3} n^{2}-n A+B & =2 n^{2}+\frac{2 q-3}{2}\left[-n+\frac{1}{3}+\frac{q-2}{2}\right] \\
& =\frac{(2 q-3)(3 q-6 n+4)+8 n^{2}}{12} .
\end{aligned}
$$

We can obtain the Pontryagin classes of $\alpha$ and $\gamma$ from (2.7)(2.9).

$$
\begin{aligned}
p_{j}(\alpha) & =(-1)^{j} c_{2 j}(\alpha \otimes c) \text { by definition } \\
& =(-1)^{j} \cdot 2 \cdot c_{2 j}\left(\psi_{C}^{3}-1\right) \zeta^{\prime \prime} .
\end{aligned}
$$

Since our calculations are over a suspension, namely, $S^{8 k-4 q}\left(C P^{2 q-1}\right)^{\eta^{2 n}}$, products of cohomology classes vanish. It follows from (2.7)-(2.9) that the possible nonzero classes are $p_{2 k}(\alpha), p_{2 k-1}(\alpha)$ and $p_{2 k}(\gamma)$ only. These are easily written down using the fact that

$$
c_{i} \psi_{C}^{3}(\quad)=3^{i} c_{i}(\quad)[1]
$$

and

$$
\begin{gathered}
c_{2 j}(\quad)=(-1)(2 j-1) ! \operatorname{ch}^{2 j}(\quad) \\
{\left[\begin{array}{cc}
p_{2 k}(\alpha)=(-1)(4 k-1) ! \quad\left(3^{4 k}-1\right)\left[\frac{(2 q-3-6 n-4)+8 n^{2}}{6}\right] \\
\beta^{4 k-2 q}\left(c^{2 q-1}\right) \cdot U_{\eta} 2 n \\
p_{2 k-1}(\alpha)=(4 k-3) !\left(3^{4 k-2}-1\right) \cdot 2 \cdot \beta^{4 k-2 q}\left(c^{2 q-2}\right) \cdot U^{\eta^{2 n}} \\
p_{2 k}(\gamma)=(-1)(4 k-1) !\left(3^{4 k}-1\right) \beta^{4 k-2 q}\left(c^{2 q-1}\right) \cdot U^{\eta^{2 n}}
\end{array}\right.}
\end{gathered}
$$

Here $\beta^{4 k-2 q}$ denotes the $(8 k-4 q)$-fold suspension in cohomology. We may drop $\beta^{2 k-2 q}$ and $U^{\eta^{2 n}}$ from (2.10) with no loss of clarity.

$$
\begin{aligned}
& \left\langle L_{2 k}\left(\tau_{M} \oplus 3^{e} x \alpha \oplus 3^{e} y \gamma\right),[M]\right\rangle=\left\langle L_{2 k}\left(\tau_{M}\right),[M]\right\rangle \\
& \quad+\left\langle L_{1}\left(\tau_{M}\right) L_{2 k-1}\left(3^{e} \times \alpha \oplus 3^{e} y \gamma,[M]\right\rangle\right. \\
& \quad+\left\langle L_{2 k}\left(3^{e} x \alpha \oplus 3^{e} y \gamma,[M]\right\rangle .\right.
\end{aligned}
$$

So in order to prove the lemma, we must be able to choose $x, y$ (with $y$ odd) such that $\left\langle L_{1}\left(\tau_{M}\right) L_{2 k-1}\left(3^{e} x \alpha \bigoplus 3^{e} y \gamma\right),[M]\right\rangle=-\left\langle L_{2 k}\left(3^{e} x \alpha \bigoplus\right.\right.$ $\left.\left.3^{e} y \gamma\right),[M]\right\rangle$. Again, since $\alpha, \gamma$ are defined on a suspension, we have $L_{m}=1_{m} p_{m}$ where $1_{m}=2^{2 m+1}\left(2^{2 m-1}-1\right) /(2 m-1) ! j_{4 m} \operatorname{num}\left(B_{m} / 4 m\right)$ $[8,16] j_{4 m}=$ order $J\left(S^{4 m-1}\right)=$ odd/odd $\cdot\left(3^{2 m}-1\right)$ and $\operatorname{num}\left(B_{m} / 4 m\right)$ is odd $\left(B_{m}\right.$ is the $n$th Bernoulli number [3]). Since $L_{1}\left(\tau_{M}\right)=p_{1}(M) / 3$, we want 


$$
\left\langle p_{1}(M) \cdot \frac{1}{3} \cdot 1_{2 k-1} p_{2 k-1}(x \alpha),[M]\right\rangle=\left\langle-1_{2 k}\left(p_{2 k}(x \alpha)+p_{2 k}(y \gamma),[M]\right\rangle\right.
$$

This relation can be written as

$$
y a=x b
$$

where

$$
a=-\left\langle 1_{2 k} p_{2 k}(\gamma),[M]\right\rangle=\left\langle 2^{4 k+1} \cdot \frac{\text { odd }}{\text { odd }} \cdot c^{2 q-1},[M]\right\rangle \text { from }(1.10) .
$$

We write $b=b^{\prime}-b^{\prime \prime} ; b^{\prime \prime}=\left\langle L_{2 k} p_{2 k}(\alpha),[M]\right\rangle$

$$
\begin{aligned}
& =2^{4 k+1} \cdot \frac{\text { odd }}{\text { odd }} \cdot\left[\frac{(2 q-3)(3 q-6 n+4)+8 n^{2}}{6}\right] \cdot\left\langle c^{2 q-1},[M]\right\rangle \\
& =2^{4 k+1} \cdot \frac{\text { odd }}{\text { odd }} \cdot \frac{1}{2} \cdot \frac{\text { odd }}{\text { odd }}\left[(3 q-6 n+4)+8 n^{2}\right] \cdot\left\langle c^{2 q-1},[M]\right\rangle .
\end{aligned}
$$

Therefore

$$
b^{\prime \prime}=2^{4 k} \cdot \frac{\text { odd }}{\text { odd }} \cdot\left\langle c^{2 q-1},[M]\right\rangle \text { if } q \text { is nonzero } \bmod 4 \text { and } n \text { is }
$$

$$
\text { even or } q \text { is zero } \bmod 4 \text { and } n \text { is odd. }
$$

$b^{\prime}=\left\langle\frac{p_{1}(M)}{3} 1_{2 k-1} p_{2 k-1}(\alpha),[M]\right\rangle$. To calculate $b^{\prime}$ we need $p_{1}(M)$.

$M=S^{8 k-4 q+2} \times{ }_{S^{1}} S^{4 q-1} \stackrel{\Pi}{\longrightarrow} C P^{2 q-1}$ be the bundle (trivial $\bigoplus \eta^{2 n}$ ).

$\tau_{M}=\Pi^{*} \tau C P^{2 q-1} \oplus$ the tangent along the fibers, is stably equal to $\Pi^{*} \tau^{C P^{2 q-1}} \oplus \Pi^{*} \eta^{2 n}$. Hence, $p_{1}\left(\tau_{M}\right)=2 q \Pi^{*} c^{2} \pm 4 n^{2} \Pi^{*} c^{2}=\left(2 q \pm 4 n^{2}\right) \Pi^{*} c^{2}$. Substituting this value of $p_{1}(M)$ in $b^{\prime}$, we see that

$$
b^{\prime}=2^{4 k} \cdot \frac{\text { even }}{\text { odd }} \cdot\left\langle c^{2 q-1},[M]\right\rangle
$$

and

$$
b=b^{\prime}-b^{\prime \prime}=2^{4 k} \cdot \frac{\text { odd }}{\text { odd }} \cdot\left\langle c^{2 q-1},[M]\right\rangle
$$

if $q$ is nonzero $\bmod 4$ and $n$ is even or if $q$ is zero $\bmod 4$ and $n$ is odd. It is clear from (2.11)-(2.14) that there are integers $(x, y)$, $y$ odd such that $y a=x b$. This proves the lemma.

3. Semi-free actions. We discuss semi-free circle actions on homotopy spheres not bounding spin manifolds with standard spheres as fixed point sets. The fixed point set has co-dimension $4 q$ [15]. 
G. Bredon has shown that for every $k$ there is a $8 k+2$ homotopy sphere not bounding a spin manifold which admits a smooth semifree action of the circle group [6], and in [16] Schultz proved the following complementary result in dimension $8 k+1$ :

Theorem 3.1 [16]. Let $k>0$ and $q<2 k+1$. Assume that $q$ is not equal to 1 and not equal to zero $\bmod 4$. Then there is a semi-free action of the circle on some homotopy $(8 k+1)$ - sphere not bounding a spin manifold whose fixed point set is an ordinary sphere of co-dimension $4 q$.

Levine has shown that co-dimension 4 is not realizable [12]; we wish to show that the restriction on $q$ may be relaxed in general and may be removed in low dimensional cases.

TheOREM 3.2. Let $k>0$ and $q<2 k+1$. An ordinary $(8 k-4 q+1)-$ sphere can be realized as the fixed point set of a smooth semi-free circle action on a homotopy $(8 k+1)$-sphere not bounding a spin manifold provided $q$ is not equal to $0 \bmod 32$.

Our arguments closely follow those in [16] and the first part of this paper. First we establish an analogue of Lemma 2.3. Let

$$
\begin{aligned}
s & =q-1 \\
M & =S^{8 k-4 q+2} \times C P^{2 s+1} \\
\alpha & =\psi_{R}^{3} r e \beta^{4 k-2 s-1}\left(\mu^{2 s-3}\right) \\
& =\psi_{R}^{3} \sigma-\sigma, \text { where } \sigma \otimes C \text { generates } K\left[S^{8 k-4 s-2}\left(\frac{C P^{2 s+1}}{C P^{2 s}}\right)\right]=K\left(S^{8 k}\right) .
\end{aligned}
$$

We can calculate the Pontryagin classes of $\alpha$ and $\gamma$ just as in Lemma 2.3. We see that

$$
p_{n}(\alpha)=(-1)^{n+1} .2 .(2 n-1) !\left(3^{2 n}-1\right) \operatorname{ch}^{2 n} \beta^{8 k-4 s-2}\left(\mu^{2 s-3}\right)
$$

and

$$
\operatorname{ch}\left(\mu^{2 s-3}\right)=\left(e^{c}-1\right)^{2 s-3}=c^{2 s-3}+A^{\prime} c^{2 s-1}+B^{\prime} c^{28+1}+\text { terms }
$$

of even degree in c. Here

$$
\begin{aligned}
& A^{\prime}=\frac{2 s-3}{6}+\frac{1}{4}\left(\begin{array}{c}
2 s-3 \\
2
\end{array}\right) \\
& B^{\prime}=\frac{2 s-3}{5 !}+\left(\begin{array}{c}
2 s-3 \\
2
\end{array}\right)\left[\frac{1}{4 !}+\frac{1}{(3 !)^{2}}\right]+\frac{1}{8}\left(\begin{array}{c}
2 s-3 \\
3
\end{array}\right)+\frac{1}{16}\left(\begin{array}{c}
2 s-3 \\
4
\end{array}\right) .
\end{aligned}
$$

Therefore, the only nonzero Pontryagin classes are $p_{2 k}(\alpha), p_{2 k-1}(\alpha)$ 
and $p_{2 k-2}(\alpha)$. These are given by

$$
\begin{aligned}
& p_{2 k}(\alpha)=(-2)(4 k-1) !\left(3^{4 k}-1\right) B^{\prime} c^{2 s-1} \\
& p_{2 k-1}(\alpha)=(2)(4 k-3) !\left(3^{4 k-2}-1\right) A^{\prime} c^{2 s-2} \\
& p_{2 k-2}(\alpha)=(-2)(4 k-5) !\left(3^{4 k-4}-1\right) c^{2 s-3}
\end{aligned}
$$

Note that the right sides have to be suspended enough times to place them in the proper cohomology groups.

As in Lemma 2.2, $\sigma \otimes C=\beta^{4 k-2 s-1}\left(\mu^{2 s+1}\right)$ and hence

$$
p_{2 k}(\gamma)=(-1)(4 k-1) !\left(3^{4 k}-1\right) c^{2 s+1}
$$

and $p_{i}(\gamma)=0$ if $i \neq 2 k$. Also note that

$$
\begin{aligned}
& L_{1}\left(C P^{2 s+1}\right)=\frac{1}{3} p_{1}\left(C P^{2 s+1}\right)=\frac{2}{3}(s+1) \\
& L_{2}\left(C P^{2 s+1}\right)=\frac{1}{45}\left(7 p_{2}-p_{1}^{2}\right)=\frac{1}{45}(s+1)(10 s-3)
\end{aligned}
$$

$\alpha, \gamma$ can be considered to be bundles over $M ; 3^{e} \alpha, 3^{e} \gamma$ are therefore elements of $[M, F / 0]$ for large values of $e$. We have the following proposition

Proposition 3.8. There are integer pairs $(x, y)$ such that $y$ is odd and $\left\langle L_{2 k}\left(\tau_{M} \oplus 3^{e} x \alpha \oplus 3^{e} y \gamma\right),[M]\right\rangle=0$, provided $q$ is even and nonzero $\bmod 32$.

Proof of Theorem 3.2. Let $(x, y)$ be an integer pair given by Proposition 3.8. $3^{e} x \alpha \oplus 3^{e} y \gamma$ gives a normal map $f_{x, y}: X^{8 k} \rightarrow M$ such that

$$
\tau_{X}+f^{*}\left(\gamma_{M}\right)=f^{*}\left(3^{e} x \alpha \oplus 3^{e} y \gamma\right) .
$$

It is possible to choose $f$ such that $f / f_{x, y}^{-1}\left(D_{-}^{8 k-4 s-2} \times C P^{2 s+1}\right)$ is a diffeomorphism. Excising the manifold on which $f$ is a diffeomorphism, we get a relative map $f_{x, y}^{\prime}:(X, \partial X) \rightarrow\left(D^{8 k-4 s-2}, S^{8 k-4 s-3}\right) \times C P^{2 s+1}$ such that $f^{\prime} / \partial X$ is a diffeomorphism. The relative surgery obstruction of $f^{\prime}$ is the same as the surgery obstruction of $f$ since all spaces involved are simply connected [20]. So $f^{\prime}$ is normally co-bordant to a homotopy equivalence if and only if surgery obstruction of $f=1 / 8[$ Index $X$-Index $M]=0=\operatorname{Index} M$, since Index $M=0$. But

$$
\begin{aligned}
\text { Index } X=\left\langle L_{2 k} \tau_{X},[X]\right\rangle & =\left\langle L_{2 k} f^{*} \tau_{M} \oplus 3^{e} x \alpha \oplus 3^{e} y \gamma,[X]\right\rangle \\
& =\left\langle L_{2 k} \tau_{M} \oplus 3^{e} x \alpha \oplus 3^{e} y \gamma,[M]\right\rangle .
\end{aligned}
$$

This last expression is zero by Proposition 3.8 and our choice of $x$ 
if $q$ is even. So $f^{\prime}$ is normally co-bordant to a homotopy equivalence which is a diffeomorphism on the boundary. Consider the pullback diagram

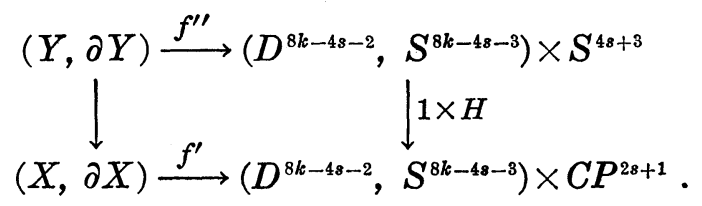

Here $H$ is the Hopf fiber map. The pullback $(Y, \partial Y) \rightarrow(X, \partial X)$ is a principal $S^{1}$ bundle map and hence there is a circle action on $(Y, \partial Y)$ such that $f^{\prime \prime}$ is equivariant. Further, $f^{\prime \prime}$ is a homotopy equivalence which is a diffeomorphism on $\partial Y$. Therefore $\sum_{x, y}^{8 k+1}=$ $Y U_{f^{\prime \prime}, \partial Y} S^{8 k-4 s-3} \times D^{4 s+4}$ has a smooth effective circle action given by the actions on the component pieces. This action is semi-free and the fixed point set is $S^{8 k-4 q+1}$. By van Kampen's theorem $\sum_{x, y}^{8 k+1}$ is simply connected. A Mayer-Vietoris argument shows that it is a homology sphere. A discussion similar to the one in the proof of Theorem 2.1 shows that this sphere does not bound a spin manifold.

When $q$ is odd, the theorem is contained in [16].

REMARK 3.4. It appears from the calculations in this paper that different choices of $\alpha, \gamma$ might relax the conditions in Theorem 3.1 further. But the calculation of the surgery obstructions become unmanageably involved. Probably a transition to use of some machinery from analytical number theory is called for. The reader may be referred to [8].

4. Realizability of any ordinary sphere as a fixed point set. In this final section we assemble our results into a single main theorem. We need to state $(8 k+2)$ dimensional versions of Theorems 2.1, 3.2.

THEOREM 4.1. Let $k>0$. There is a smooth effective circle action on the homotopy $(8 k+2)$-sphere not bounding a spin manifold with fixed point set a standard sphere of codimension $4 q+2, q>1$.

THEOREM 4.2. Let $k>0, q<2 k+1$ and $q$ not equal to 1. Any ordinary sphere $S^{8 k-4 q+2}$ can be realized as the fixed point set of a smooth semi-free action of the circle group on a homotopy $(8 k+2)$ sphere not bounding a spin manifold.

These results were known to Bredon [6]. The following, then, summarises our results. 
MaIN Theorem 4.3. Any ordinary sphere $S^{n}(n>0)$ can be realized as the fixed point set of an infinite number of smooth effective actions of the circle group on a $\Sigma^{8 k+1}$ or $\Sigma^{8 k+2}$ not bounding spin manifolds, for every $k>0,8 k>n-1$. These actions are distinguished by their local representation at fixed points.

Proof. The verification splits into different cases according to the value of $n$ modulo 4 .

Case (1). $n=-1$ or $0 \bmod 4$

This follows from Theorems 2.1 and 4.1. These actions are not semi.free.

Case (2). $n=1$ or $2 \bmod 4$

Theorem 4.2 gives semi-free smooth effective actions on $\Sigma^{8 k+2}$ with $S^{4 t-2}$ as fixed point sets. Theorem 3.2 gives smooth semi-free actions on $\sum^{8 k+1}$ with $S^{4 t+1}$ as fixed point sets provided $2 k-2$ is nonzero mod 32 . We are not able to remove this provision keeping the actions semi-free. However, there is no difficulty in obtaining the spheres in Case (2) as fixed point sets of nonsemi-free smooth effective actions on $\Sigma^{8 k+1}, \Sigma^{8 k+2}$ not bounding spin manifolds. Computations identical to those in $\S 2$ show that it is enough to consider actions with local representation [trivial $\oplus \psi^{3 n} C \oplus(2 q-1) C$ ].

Final Remarks. The author wishes to thank Prof. Schultz for introducing him to the subject and for his constant encouragement.

The suggestions of the referee have made this article better organized. In particular, $\S 1$ was expanded and rewritten according to his suggestion. The author gratefully acknowledges the referee's advice.

\section{REFERENCES}

1. J. F. Adams, Vector fields on spheres, Ann. of Math., (2) 75 (1962), 603-632.

2. - On the groups $J(X) I$, Topology, 2 (1963).

3. - On the groups $J(X) I V$, Topology, 5 (1966).

4. J. F. Adams and J. Walker, On complex Steifel manifolds, Proc. of the Cambridge Philos. Soc., 61 (1965), 81-103.

5. D. W. Anderson, E. H. Brown and F. P. Peterson, The structure of the spin cobordism ring, Ann. of Math., (2) 86 (1967), 271-298.

6. G. Bredon, $A \Pi_{*}$ module structure for $\Theta_{*}$ and applications to transformation groups, Ann. of Math., (2) 86 (1967), 434-448.

7. W. Browder, Surgery on simply connected manifolds, Ergebnisse der Mathematic und ihrer Granzgebeite, Band 65, Springer Verlag, New York, 1972.

8. M. Arkowitz, J. Ewing and S. Schiffman, $H$ structures on localized and completed spheres, Quart. Journal of Math., 26 (1975), 295-307.

9. J. Becker and R. Schultz, Equivariant function spaces and stable homotopy theory I, Comm. Math, Helvitici, 49 (1974), 1-34.

10. S. Feder and S. Gitler, Stunted projective spaces and the J-order of the Hopf 
bundle, Bull. Amer. Math. Soc., 80 (1974), 748-749.

11. S. Feder and S. Gitler, The classification of stunted projective spaces by stable homotopy type, Trans. Amer. Math. Soc., 225 (1977), 59-81.

12. J. Levine, Semi-free circle actions on spheres, Invent. Math., 22 (1973), 161-186.

13. D. Quillen, The Adams Conjecture, Topology, 10 (1970), 67-80.

14. R. Schultz, Smooth actions of small groups on exotic spheres, Proc. Symp. Pure Math., 32 (1978), 491-496.

15. - The nonexistence of semi-free $S^{1}$ actions on homotopy spheres, Proc. Amer. Math. Soc., 27 (1971), 595-597.

16. Circle actions on homotopy spheres not bounding spin manifolds, Trans. Amer. Math. Soc., 213 (1975), 89-98.

17. - Differentiable group actions on homotopy spheres I, Invent. Math., 31 (1975), 105-128.

18. D. Sullivan, Geometric Topology I, Localization, Periodicity, and Galois Symmetry, Mimeographed: Massachusetts Institute of Technology, 1970.

19. Genetics of homotopy theory, Ann. of Math., 100 (1974).

20. C. T. C. Wall, Surgery on compact manifolds, London Math. Soc. Monographs No. 1, Academic Press, New York, 1970.

21. R. Schultz, Differentiable group actions on homotopy spheres $I V$, to appear.

Received April 10, 1979.

N. C. A. ANd T. State University

GREENSBORO, NC 27411 


\section{PACIFIC JOURNAL OF MATHEMATICS}

\section{EDITORS}

DONALD BABBITT (Managing Editor)

University of California

Los Angeles, CA 90024

HUGo RossI

University of Utah

Salt Lake City, UT 84112

C. C. MOORE and ANDREW OGG

University of California

Berkeley, CA 94720
J. DugundjI

Department of Mathematics

University of Southern California

Los Angeles, CA 90007

R. FinN and J. Milgram

Stanford University

Stanford, CA 94305

\section{ASSOCIATE EDITORS}
R. ARENS
E. F. BECKENBACH
B. H. NeumanN
F. WOLF
K. YOSHIDA

\section{SUPPORTING INSTITUTIONS}

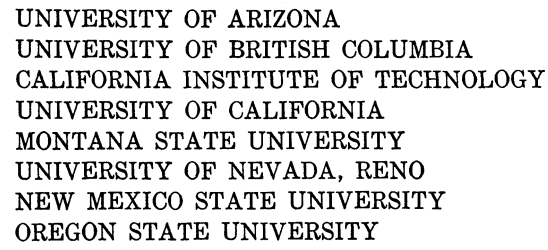

UNIVERSITY OF ARIZONA

UNIVERSITY OF BRITISH COLUMBIA

CALIFORNIA INSTITUTE OF TECHNOLOGY

UNIVERSITY OF CALIFORNIA

MONTANA STATE UNIVERSITY

UNIVERSITY OF NEVADA, RENO

NEW MEXICO STATE UNIVERSITY

OREGON STATE UNIVERSITY

\author{
UNIVERSITY OF OREGON \\ UNIVERSITY OF SOUTHERN CALIFORNIA \\ STANFORD UNIVERSITY \\ UNIVERSITY OF HAWAII \\ UNIVERSITY OF TOKYO \\ UNIVERSITY OF UTAH \\ WASHINGTON STATE UNIVERSITY \\ UNIVERSITY OF WASHINGTON
}

The Supporting Institutions listed above contribute to the cost of publication of this Journal, but they are not owners or publishers and have no responsibility for its content or policies.

Mathematical papers intended for publication in the Pacific Journal of Mathematics should be in typed form or offset-reproduced, (not dittoed), double spaced with large margins. Please do not use built up fractions in the text of the manuscript. However, you may use them in the displayed equations. Underline Greek letters in red, German in green, and script in blue. The first paragraph or two must be capable of being used separately as a synopsis of the entire paper. Please propose a heading for the odd numbered pages of less than 35 characters. Manuscripts, in triplicate, may be sent to any one of the editors. Please classify according to the scheme of Math. Reviews, Index to Vol. 39. Supply name and address of author to whom proofs should be sent. All other communications should be addressed to the managing editor, or Elaine Barth, University of California, Los Angeles, California, 90024.

50 reprints to each author are provided free for each article, only if page charges have been substantially paid. Additional copies may be obtained at cost in multiples of 50 .

The Pacific Journal of Mathematics is issued monthly as of January 1966. Regular subscription rate: $\$ 102.00$ a year (6 Vols., 12 issues). Special rate: $\$ 51.00$ a year to individual members of supporting institutions.

Subscriptions, orders for numbers issued in the last three calendar years, and changes of address shoud be sent to Pacific Journal of Mathematics, P.O. Box 969, Carmel Valley, CA 93924, U.S.A. Old back numbers obtainable from Kraus Per!odicals Co., Route 100, Millwood, NY 10546.

PUBLISHED BY PACIFIC JOURNAL OF MATHEMATICS, A NON-PROFIT CORPORATION

Printed at Kokusai Bunken Insatsusha (International Academic Printing Co., Ltd.). 8-8, 3-chome, Takadanobaba, Shinjuku-ku, Tokyo 160, Japan. 


\section{Pacific Journal of Mathematics}

\section{Vol. 95, No. $2 \quad$ October, 1981}

George E. Andrews, The Rogers-Ramanujan reciprocal and Minc's

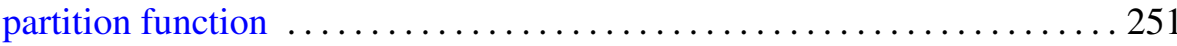

Allan Calder, William H. Julian, Ray Mines, III and Fred Richman,

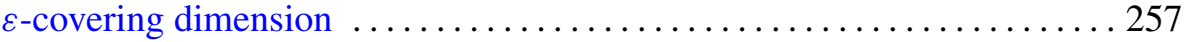

Thomas Curtis Craven and George Leslie Csordas, An inequality for the distribution of zeros of polynomials and entire functions $\ldots \ldots \ldots \ldots 263$

Thomas Jones Enright and R. Parthasarathy, The transfer of invariant

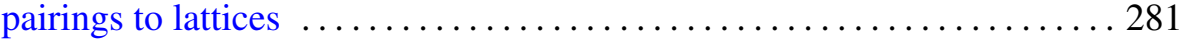

Allen Roy Freedman and John Joseph Sember, Densities and

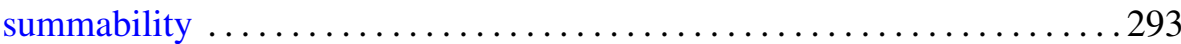

Robert Heller and Francis Aubra Roach, A generalization of a classical necessary condition for convergence of continued fractions . . . . . . 307

Peter Wilcox Jones, Ratios of interpolating Blaschke products ........... 311

V. J. Joseph, Smooth actions of the circle group on exotic spheres ........ 323

Mohd Saeed Khan, Common fixed point theorems for multivalued

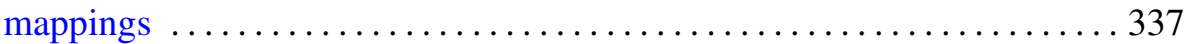

Samuel James Lomonaco, Jr., The homotopy groups of knots. I. How to

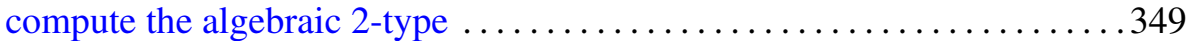

Louis Magnin, Some remarks about $C^{\infty}$ vectors in representations of connected locally compact groups ............................ 391

Mark Mandelker, Located sets on the line . . . . . . . . . . . . . . . . . 401

Murray Angus Marshall and Joseph Lewis Yucas, Linked quaternionic mappings and their associated Witt rings $\ldots \ldots \ldots \ldots \ldots \ldots \ldots \ldots . \ldots \ldots 11$

William Lindall Paschke, $K$-theory for commutants in the Calkin algebra

W. J. Phillips, On the relation $P Q-Q P=-i I$ 435

Ellen Elizabeth Reed, A class of Wallman-type extension. 443

Sungwoo Suh, The space of real parts of algebras of Fourier transforms 461 Antonius Johannes Van Haagen, Finite signed measures on function

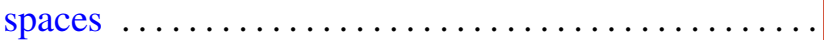

Richard Hawks Warren, Identification spaces and unique uniformity 